\title{
Table-Top X-ray Spectroscopy of Benzene Radical Cation
}

\author{
Michael Epshtein ${ }^{1,2}$, Valeriu Scutelnic ${ }^{1}$, Zheyue Yang ${ }^{1}$, Tian Xue ${ }^{1}$, Marta L. Vidal ${ }^{3}$, Anna I. Krylov ${ }^{4}$, \\ Sonia Coriani ${ }^{3}$, and Stephen R. Leone $e^{1,2,5^{*}}$ \\ ${ }^{1}$ Department of Chemistry, University of California, Berkeley, CA, 94720, USA \\ ${ }^{2}$ Chemical Sciences Division, Lawrence Berkeley National Laboratory, Berkeley, CA, 94720, USA \\ ${ }^{3}$ DTU Chemistry - Department of Chemistry, Technical University of Denmark, DK-2800, \\ Kongens Lyngby, Denmark \\ ${ }^{4}$ Department of Chemistry, University of Southern California, Los Angeles, California \\ 90089, USA \\ ${ }^{5}$ Department of Physics, University of California, Berkeley, CA, 94720, USA
}

\begin{abstract}
Ultrafast table-top x-ray spectroscopy at the carbon K-edge is used to measure the x-ray spectral features of benzene radical cations $\left(\mathrm{Bz}^{+}\right)$. The ground state of the cation is prepared selectively by two-photon ionization of neutral benzene, and the $\mathrm{x}-$ ray spectra are probed at early times after the ionization by transient absorption using $\mathrm{x}$-rays produced by high harmonic generation (HHG). Bz ${ }^{+}$is well known to undergo Jahn-Teller distortion, leading to a lower symmetry and splitting of the $\pi$ orbitals. Comparison of the x-ray absorption spectra of the neutral and the cation reveals a splitting of the two degenerate $\pi^{*}$ orbitals as well as an appearance of a new peak due to excitation to the partially occupied $\pi$-subshell. The $\pi^{*}$ orbital splitting of the cation, elucidated on the basis of high-level calculations in a companion theoretical paper [Vidal et al, submitted to J. Phys. Chem. Lett.; ChemRxiv link: doi XXXXX], is discovered to be due to both the symmetry distortion and even more dominant spin coupling of the unpaired electron in the partially vacant $\pi$ orbital (from ionization) with the unpaired electrons resulting from the transition from the $1 \mathrm{sc}$ core orbital to the fully vacant $\pi^{*}$ orbitals.
\end{abstract}

\section{INTRODUCTION}

The recent development of tabletop HHG sources reaching up to $300 \mathrm{eV}$ photon energies in the $\mathrm{x}$-ray ${ }^{1,2,3,4,5,6,7,8,9,10}$ gives rise to a powerful pump-probe technique that combines near-infrared (NIR) or ultraviolet (UV) excitation and soft $\mathrm{x}$-ray spectroscopic probing in the carbon K-edge region. Promotion of an electron from the carbon K-edge 1 s orbitals to vacant or partially vacant valence orbitals following NIR/UV excitation or ionization provides the opportunity to follow ultrafast electronic structural changes via the x-ray spectroscopic regime. ${ }^{11,12,13}$

$\mathrm{X}$-ray spectroscopy is very sensitive to the changes in molecular structure and orbital occupancy, giving accurate new information about energetics and dynamics of transient species. Taking advantage of the typical wide spectral range of the HHG flux, lightinitiated chemical reactions in the lowest electronic states of isolated $\mathrm{CF}_{4}{ }^{+}$and $\mathrm{SF}_{6}{ }^{+}$molecules were studied in the carbon K-edge and sulfur L-edge regions, respectively. The reaction paths were characterized and the effect of symmetry breaking was observed through the splitting of absorption bands and Rydberg-valence mixing induced by the geometry changes. ${ }^{14}$ In parallel, the pericyclic minimum that leads to the ultrafast ring-opened product or the ground state reformation of 1,3-cyclohexadiene was characterized. ${ }^{15}$ Dynamics of ring opening in furfural following $267 \mathrm{~nm}$ excitation was also observed, ${ }^{16}$ as well as intersystem crossing in acetylacetone ${ }^{17}$ and additional studies in the carbon K-edge region. ${ }^{18,19}$ Even at higher energies like oxygen K-edge, ultrafast dissociation of water cation ${ }^{20}$ and formation of the $\mathrm{OH}$ radical have been probed..$^{21} \mathrm{X}$-ray spectroscopy can be also applied to probe excited states of cations, which are difficult to observe with UV or visible spectroscopy. This advantage is exploited in the present investigation to probe the electronic structure of the ground-state benzene cation, its unoccupied orbitals and the partially vacant $\pi$ orbitals, which are known to be affected by Jahn-Teller (JT) distortion. ${ }^{22,23,24}$ The ground electronic state of benzene, $\tilde{X}^{2} E_{1 g}$, has two $\pi\left(\mathrm{e}_{\mathrm{g}}\right)$ degenerate highest occupied molecular orbitals (HOMO) and two $\pi^{*}\left(\mathrm{e}_{2 \mathrm{u}}\right)$ degenerate lowest unoccupied molecular orbitals (LUMO). Removing an electron from one of the degenerate $\pi$ orbitals leads to electronic states of the cation: $\mathrm{Bz}^{+}\left(\mathrm{B}_{2 \mathrm{~g}}\right)$ and $\mathrm{Bz}^{+}\left(\mathrm{B}_{3 \mathrm{~g}}\right)$. The geometric relaxation of the cation splits the electronic degeneracy between the orbitals (and the states) and results in two nearly degenerate minima (compressed and elongated structures) connected via a small barrier, well below the zero-point energy. ${ }^{22}$ From ro-vibrational spectroscopy and previous calculations it is known that the energetic difference between the two JT structures is very small, ${ }^{25,26}$ approximately $0.002 \mathrm{eV}$, much smaller than the spectral broadening due to the short lifetime of the core level $(\sim 0.1 \mathrm{eV})$ as well as the experimental resolution $(\sim 0.3 \mathrm{eV})$ in the laboratory table-top setup. Therefore, dynamically $\mathrm{Bz}^{+}$behaves as one scrambled structure, ${ }^{25}$ which can be represented by just one electronic state, while the effect of the two JT structures will be mentioned later.

In $\mathrm{Bz}^{+}$, the energetic degeneracy of the two $\pi\left(\mathrm{e}_{\mathrm{gg}}\right)$ orbitals is lifted due to symmetry lowering, and the same is expected to occur in the virtual $\pi^{*}$ orbitals (LUMO); a similar effect was described in core-ionized benzene. ${ }^{27,28}$ In addition, each of the $1 \mathrm{~s} \rightarrow \pi^{*}$ transitions can be split due to spin coupling in the presence of the partially vacant $\pi$ orbital, as explained in the companion theoretical 
paper. ${ }^{29}$ Analogous splittings were recently reported in $\mathrm{N}_{2}{ }^{+30}$ as well as at the oxygen $\mathrm{K}$-edge of $\mathrm{CO}^{+}{ }^{31} \mathrm{In} \mathrm{N}_{2}{ }^{+}$, the features at 402.2 $\mathrm{eV}$ and $403.1 \mathrm{eV}$ correspond to the $\pi^{*}$ excitation, as assigned through x-ray absorption spectroscopy (XAS) of neutral nitrogen molecules, but with a $3 \sigma_{\mathrm{g}}$ spectator hole in the case of the ion, i.e., a $3 \sigma_{\mathrm{g}}{ }^{-1}{ }^{2} \Sigma_{\mathrm{g}} \rightarrow 1 \sigma_{\mathrm{u}}{ }^{-1} 3 \sigma_{\mathrm{g}}{ }^{-1} 1 \pi_{\mathrm{g}}{ }^{2} \Pi_{\mathrm{u}}$ transition. There is a splitting of the $1 \sigma_{\mathrm{u}}^{-1} 3 \sigma_{\mathrm{g}}{ }^{-1} 1 \pi_{\mathrm{g}}{ }^{2} \Pi_{\mathrm{u}}$ state, due to two possible spin couplings. Different spin couplings of three unpaired electrons arise from spin adaptation of the wave function and have been discussed in detail. $^{32,33}$

This paper experimentally investigates the influence of spin couplings and JT distortion by probing benzene cations with broad $\mathrm{x}$ ray radiation in the carbon $\mathrm{K}$-edge region and comparing to the $\mathrm{x}$ ray spectra of neutral benzene. Specifically, we probe the electronic transitions from the core to the partially vacant $\pi$ orbital (produced by ionization) as well as to the vacant $\pi^{*}$ orbitals of the benzene cations. In addition, we discuss in detail the preparation of the cation by two-photon ionization in the ultraviolet, leading to relatively cold (minimal excitation above room temperature thermal energy) benzene cation on the electronic ground state, probed by $\mathrm{x}$-ray spectroscopy, and examine the relaxation dynamics from the Franck-Condon (FC) region of the neutral to the relaxed cation in a time-dependent experiment. The complete analysis of the experimental spectra using high-level ab initio calculations is presented in the companion theoretical paper. ${ }^{29}$

\section{METHODS}

The experimental setup has been described elsewhere. ${ }^{15}$ The experiments were carried out with broadband soft x-ray pulses (extending to $\sim 360 \mathrm{eV}$, see Figure 1(e) and Figure S1) that probe the ground state of $\mathrm{Bz}$ and $\mathrm{Bz}^{+}$following two-photon ionization with

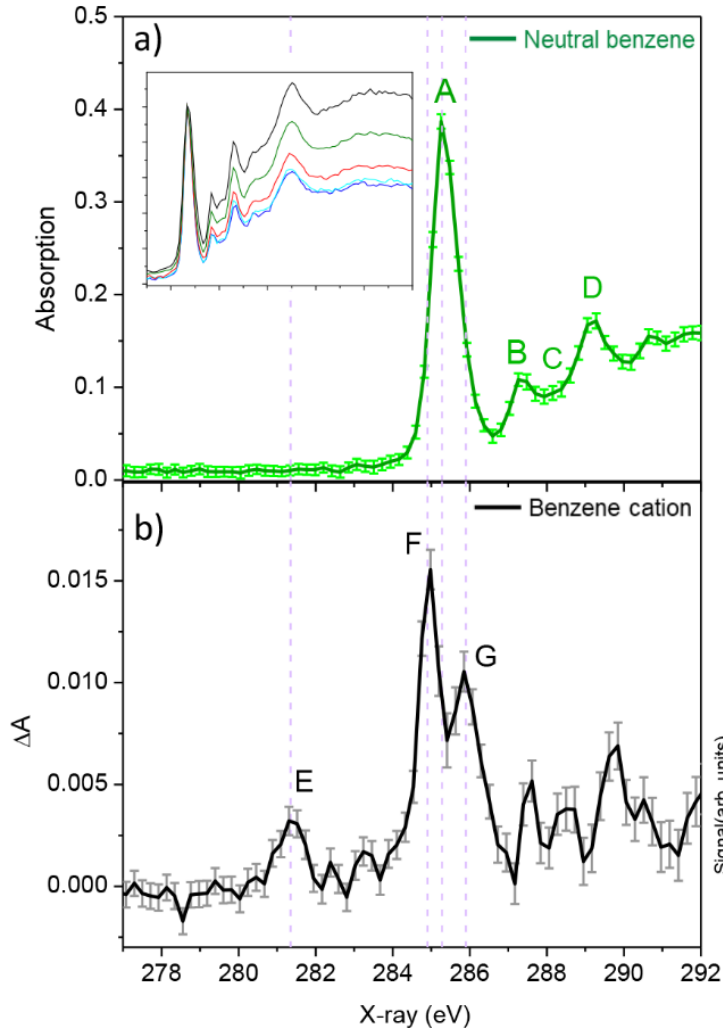

$267 \mathrm{~nm}$ light. $90 \%$ of the output of a Ti:sapphire laser that delivers $12 \mathrm{~mJ}$ pulses at $1 \mathrm{kHz}$ repetition rate, $35 \mathrm{fs}$ pulse duration, with a wavelength centered at $800 \mathrm{~nm}$ (Spectra-Physics, Spitfire-ACEPA) was converted in an optical parametric amplifier into mid infrared $2.5 \mathrm{~mJ}$ pulses $(1470 \mathrm{~nm})$. This pulse focused with a $30 \mathrm{~cm}$ focal length (f.l.) lens to a differentially pumped cell with helium atoms at a pressure of $\sim 1300$ Torr, to generate broadband soft $\mathrm{x}$-ray pulses in the carbon K-edge region. For more details see Figure S1. The other $10 \%$ of the $800 \mathrm{~nm}$ beam is used for the generation of the $267 \mathrm{~nm}$ ultraviolet pump pulse in nonlinear crystals $\left(25 \mu \mathrm{J}, 400 \mathrm{~cm}^{-}\right.$ ${ }^{1}$ bandwidth, $~ 90 \mathrm{fs}$ pulse duration). The $267 \mathrm{~nm}$ pulses are used to prepare benzene ions by two-photon ionization, and these are probed by the broadband, soft x-ray pulse at the carbon K-edge. The pump and probe beams are focused and overlapped at $\mathrm{a} \sim 1^{\circ}$ crossing angle into a gas cell that contains benzene at a pressure up to $\sim 25$ Torr (backing pressure; the cell has two pinholes for entrance and exit of the laser that allow flow of the gas out). After the cell, the probe pulse is spectrally dispersed by a Hitachi grating (001-0660) onto an X-ray CCD camera (Princeton Instruments, PIXIS:XO 400B), where the entire broadband soft $\mathrm{x}$-ray signal is measured at once as a function of the photon energy.

\section{RESULTS AND DISCUSSION}

We first present an overview of the spectra and then discuss in detail how the spectra of benzene cation are obtained and verified. Figure 1 shows experimental table-top x-ray absorption spectra $(A$ $=\log \left(I_{\text {without } B z} / I_{\text {with } B z}\right)$ of neutral benzene and the benzene cation ( $\triangle A$ - differential spectra with ultraviolet pump on versus pump off, with an added back percentage of neutral spectrum, see figure caption for details) in their ground states.

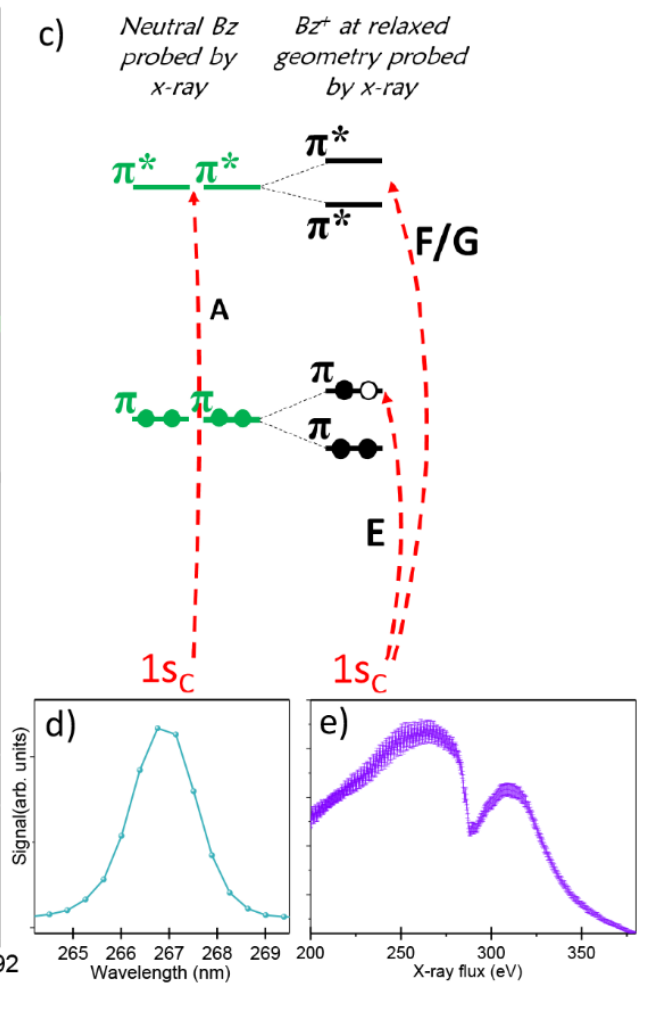

Figure 1. (a) Experimental x-ray absorption spectrum of ground-state neutral Bz presented by the green line with light green error bars corresponding to $95 \%$ confidence interval limits, the spectra obtained from A $=\log \left(I_{\text {without Bz }} / I_{\text {with Bz}}\right)$, where $I_{\text {without Bz }}$ and $I_{\text {with Bz }}$ are the transmitted signal of the broadband $\mathrm{x}$-ray flux without $\mathrm{Bz}$ molecules in the interaction region and with Bz molecules, respectively. Each spectrum is an average of 128 CCD images (1000 laser pulses per image). Inset: Raw absorption spectra of neutral benzene (normalized to the highest peak) at different pressures in the interaction region with the x-ray radiation, from highest (black line) to lowest pressure (blue line), respectively. Also $128 \mathrm{CCD}$ images averaged. (b) NEXAFS spectrum of $\mathrm{Bz}^{+}, \Delta \mathrm{A}$, taken at $0-50 \pm 50$ fs delay, represented by the black 
line with grey error bars corresponding to $95 \%$ confidence interval limits (after adding $2 \%$ of the static neutral benzene spectra, see supplemental information (SI) for details). The $\mathrm{Bz}^{+}$absorption spectrum is obtained from $\Delta \mathrm{A}=\log \left(I_{\text {without UV }} / I_{\text {with UV }}\right)$, where $I_{\text {without UV }}$ and $I_{\text {with }}$ UV are the transmitted signal of the x-ray flux without UV and with UV to ionize the molecule by two-photon ionization, respectively. The ionization UV beam is focused with a $45 \mathrm{~cm}$ focal length lens. The spectrum is an average of 512 CCD images (1000 laser pulses per image). The wavelength calibrations of the spectra are done with absorption spectra of argon and allyl radical, the calibration procedure is described in more detail in the SI. (c) Molecular orbital diagram illustrating the main transitions responsible for the dominant spectral features in the cation. Peak E corresponds to $1 \mathrm{sc} \rightarrow \pi$ transition and peaks $\mathrm{F}$ and $\mathrm{G}$ to the $1 \mathrm{sc} \rightarrow \pi^{*}$ transitions. (d) The spectral wavelength scan of the UV beam (light blue line). (e) X-ray flux (purple line), see SI for details

The spectrum of neutral benzene $(\mathrm{Bz})$ presented in Figure 1(a) is taken with a flow of benzene molecules by referencing the high harmonic x-ray flux with no sample in the cell, as previously described $^{15}$; the spectrum matches well with previously reported results, ${ }^{34,35,36,37,38,39}$ and it consists of four main peaks (labeled as AD) below the $1 \mathrm{sc}$ core level ionization energy $(290.37 \mathrm{eV}) .^{40,41} \mathrm{The}$ first peak at $285.2 \mathrm{eV}$, labeled as $\mathrm{A}$, is the most intense feature and is known as the transition from the $1 \mathrm{sc}_{\mathrm{C}}$ orbital to the $\pi^{*}\left(\mathrm{e}_{2 \mathrm{u}}\right) \mathrm{LU}$ $\mathrm{MOs},{ }^{42}$ as can be seen in the energy diagram in Figure 1(c). Peaks $\mathrm{B}, \mathrm{C}$ and $\mathrm{D}$ are due to core-to-Rydberg transitions, as also discussed in detail in the companion theoretical paper, ${ }^{29}$ which corrects a previously debated assignment of peak $\mathrm{D}$ as a transition to a higher $\pi^{*}\left(\mathrm{~b}_{1 \mathrm{~g}}\right)$ orbital, ${ }^{35,38,39,43}$ to the new assignment of doubly degenerate transitions to $\mathrm{Ry}(\mathrm{s})$ and $\mathrm{Ry}(\mathrm{p})$ character.

Special care was taken to obtain an unsaturated absorption spectrum of neutral benzene. The high vapor pressure of benzene $(\sim 100$ Torr), large absorption cross section at the carbon K-edge, and the low flux per camera pixel of the $\mathrm{x}$-ray radiation make it easy to reach a saturated regime on strong features, where nearly all photons at a specific x-ray energy are absorbed. When the peak structure is broadened due to the system's spectral resolution, the detection of saturation becomes nontrivial, since the observed peak is an average of the saturated signal and nonsaturated signal (for example, peak A consists of several vibrational states with different strengths $)^{38}$. This leads to a broader $285.2 \mathrm{eV}$ band on the highenergy side due to the saturated vibrational structure, and to a different ratio between the observed band and the core ionization region at higher energy. In this experiment, to avoid the saturated regime, the pressure is reduced until the ratio between the main peak $\mathrm{A}$ and the broad ionization region becomes constant, as shown by blue and light blue spectra in the inset of Figure 1(a), and described in more detail in the SI.

The black line in Figure 1(b) shows the absorption spectrum of $\mathrm{Bz}^{+}, \Delta A$, prepared by $267 \mathrm{~nm}$ two-photon ionization and probed by the broadband HHG pulse near the carbon K-edge (spectrum of HHG presented in Figure 1(e)) after 0-50 fs time delay. Two-photon $267 \mathrm{~nm}$ (the spectral profile presented in Figure 1(d)) excitation is in resonance with the first ionized state in which one electron is removed from a $\pi$ orbital, creating a hole in the doubly degenerate HOMO. Consequently, the lowest-energy core-level transition in the cation is the $1 \mathrm{sc}_{\mathrm{C}} \rightarrow \pi$, and the assignment of peak $\mathrm{E}$ at $281.3 \mathrm{eV}$ as $1 \mathrm{sC} \rightarrow \pi$ is straightforward.

The next transition, peak $\mathrm{F}$ at $285 \mathrm{eV}$, is assigned to $1 \mathrm{sc} \rightarrow \pi^{*}$ (LUMO), and it is red-shifted by $0.25 \mathrm{eV}$ relative to peak $\mathrm{A}$ of the neutral Bz. Since the two-photon ionization process is performed with $267 \mathrm{~nm}\left(E_{2 \mathrm{ph}}=9.29 \pm 0.04 \mathrm{eV}\right)$, benzene cations are prepared in the electronic ground state (ionization energy $\mathrm{IE}=9.243 \mathrm{eV}$ ) ${ }^{44}$ with an internal energy of approximately $0.05 \mathrm{eV}$, and there is internal vibrational energy of approximately $0.05 \mathrm{eV}$ in the room temperature benzene neutral (the vibrational population and thermal vibrational energy according to a Boltzmann distribution are shown in the SI). As long as the ionization process is limited to the absorption of two UV photons (discussed below and in the SI), this minimizes the possible contribution of internal vibrational excitation of the ion that could red-shift the observed peak F.

The lowest excited state in neutral Bz corresponds to the $\pi \rightarrow \pi^{*}$ transition, which is located at $4.72 \mathrm{eV} .{ }^{45}$ This energy differs from the gap between peaks $\mathrm{E}$ and $\mathrm{F}(\sim 3.85 \mathrm{eV})$ observed here for the cation, providing a rough estimate of the energy spacing between the $\pi$ and $\pi^{*}$ orbitals of the ion. The difference can be partly explained by changes in orbital energies (i.e., a decrease of the $\pi^{*}$ orbital $(0.25 \mathrm{eV})$ and an increase of the semi-occupied $\pi$ orbital in the cation, which occur as a result of the symmetry distortion) and partly by exchange interactions arising due to the spin couplings of the unpaired electrons. This exchange interactions leads to a blue shifted $1 \mathrm{sc} \rightarrow \pi^{*}$ transition too, assumed to be peak $\mathrm{G}$, as observed in $\mathrm{N}_{2}{ }^{+},{ }^{30}$ while symmetry distortion leading to change in orbital energies contributes to the structures of peaks $F$ and $G$ as well, which is discussed further in the companion theoretical paper. ${ }^{29}$

The preparation of benzene cation in the electronic ground state and the exclusion of possible contributions due to one photon excitation of neutral benzene or three photon or more electronically excited cations are important points for further discussion. It is always a challenging problem to limit an ionization process in an intense field to just two UV photons, nevertheless the two-photon ionization with $267 \mathrm{~nm}$ is known to be very effective in benzene. ${ }^{46}$ The first excited state of benzene, $\mathrm{S}_{0} \rightarrow \mathrm{S}_{1}$, is dipole forbidden and can only be accessed via vibronic coupling. ${ }^{47}$ Thus, because of the low one-photon cross-section $\left(10^{-20}-10^{-21} \mathrm{~cm}^{2} /\right.$ molecule $),{ }^{48,49}$ the probability of populating this state by one photon excitation is low, especially with $267 \mathrm{~nm}(4.64 \pm 0.02 \mathrm{eV})$, which is less than the energy of the $\mathrm{S}_{0} \rightarrow \mathrm{S}_{1}\left(\pi \rightarrow \pi^{*}\right)$ transition $(4.72 \mathrm{eV}){ }^{45}$ Therefore onephoton excitation of the neutral is expected to be negligible. Moreover, peak $\mathrm{E}$ and the red-shifted peak $\mathrm{F}$ are good indications that we observe the ground state of the cation. This pattern would only occur if one electron is removed from the $\pi$ orbital and the energies of the $1 \mathrm{sC} \rightarrow \pi *$ transition are split due to the structural distortion of the cation and exchange interactions of the three unpaired electrons. High level calculations using equation of motion coupledcluster theory adapted to core-level states, ${ }^{50}$ reported in the companion paper, ${ }^{29}$ provide additional support for the assignment of the $\mathrm{x}$-ray absorption spectrum of the ground state of the cation.

Special care was taken to eliminate the possibility of the formation of electronically excited cation states, which may occur due to three-photon excitation with the intense femtosecond UV pulses. Figure 2 shows the dependence of the $\mathrm{x}$-ray spectra of $\mathrm{Bz}^{+}$on $\mathrm{UV}$ power. A shorter f.l. lens of $20 \mathrm{~cm}$ was used to increase the power density over the $45 \mathrm{~cm}$ f.l. lens used for Figure 1. Panel (a) shows the $\mathrm{x}$-ray spectrum following $7 \mu \mathrm{J} /$ pulse UV pump; only one peak at low x-ray energy is observed and assigned to the $1 \mathrm{~s} \rightarrow \pi$ transition of the cation after two-photon ionization from the $\pi$ orbital. Absorption of three photons $(13.92 \mathrm{eV})$ leads to resonant ionization from the $\widetilde{D}^{2} E_{1 u}$ state $^{51}$ (producing a hole in the $\sigma_{\mathrm{C}-\mathrm{H}}$ orbital) and fast relaxation to either the ground cationic state, $\tilde{X}^{2} E_{1 g}$ with the hole in the $\pi$ HOMO, or to the $\tilde{B}^{2} E_{2 g}$ state $52,53,54$ with the hole in $\sigma_{\mathrm{C}-\mathrm{H}}$ orbital (HOMO-1). The latter is expected to be observed in the $\mathrm{x}$-ray spectrum at the lowest energy. This behavior is assigned as peaks $\mathrm{E}^{*}\left(1 \mathrm{~s}_{\mathrm{C}} \rightarrow \sigma_{\mathrm{C}-\mathrm{H}}\right)$ and $\mathrm{E}\left(1 \mathrm{~s}_{\mathrm{C}} \rightarrow \pi\right)$ in Figure $2(\mathrm{~b})$, in the $\mathrm{x}$-ray absorption spectrum following $30 \mu \mathrm{J}$ UV pump energy, and this characteristic is still observed in Figure 2(c) at $40 \mu \mathrm{J} \mathrm{UV} \mathrm{pump} \mathrm{en-}$ ergy. In addition to the appearance of peak $\mathrm{E}^{*}$, the amplitude ratios of peaks $\mathrm{E}^{*}$ and $\mathrm{E}$ compared to $\mathrm{F}$ are reduced by almost two times compared to peaks $\mathrm{E}$ and $\mathrm{F}$ in panel (a). Based on these results, we exclude three-photon ionization when the UV photon density is 
very low, and this was achieved by working with low UV power or the $45 \mathrm{~cm}$ f.l. lens for the UV laser beam as presented in Figure $1(b)$.

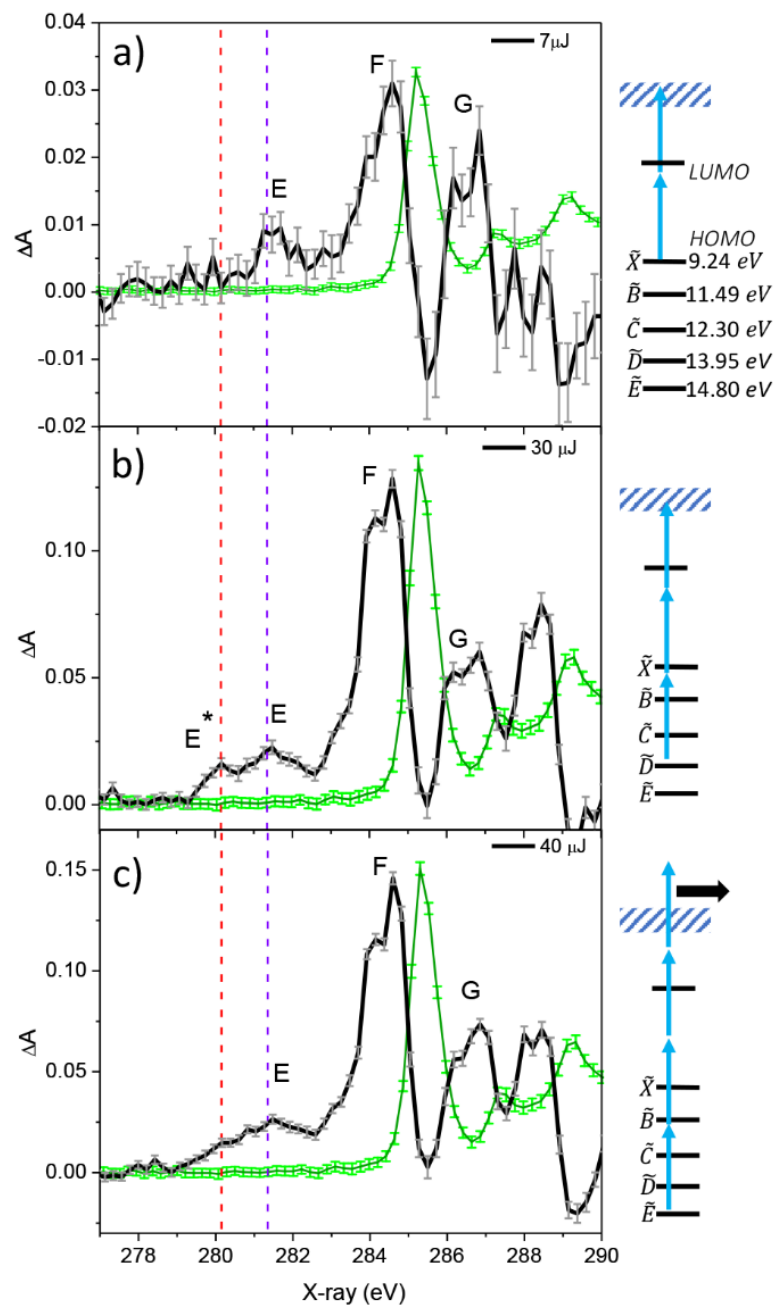

Figure 2. Comparison between $\mathrm{x}$-ray absorption spectra of $\mathrm{Bz}^{+}$with different UV powers with a $20 \mathrm{~cm}$ f.l. lens to increase the UV power density (a). $7 \mu \mathrm{J}$, (b) $30 \mu \mathrm{J}$, (c) $40 \mu \mathrm{J}$. The spectra are measured at 1 ps time delays between the pump and probe beams. The neutral benzene is presented by green lines and normalized to the main peak of $\mathrm{Bz}^{+}$. The energies in the right side of panel (a) taken from Ref ${ }^{51}$.

When the excitation energy is below $14 \mathrm{eV}$, the dissociation processes of the benzene cation are negligible. ${ }^{55}$ However, by increasing the UV power, the probability of four-photon ionization and consequently the opening of additional dissociation channels becomes greater. The signal at lower energies of the various cation or photofragments is broadened, as observed in the two lower energy peaks $(280.1$ and $281.3 \mathrm{eV}$ ) in panel 2(c) achieved by $40 \mu \mathrm{J} \mathrm{UV}$ pump. Panels (b) and (c) differ from panel (a) in the strongly increasing peak at $288 \mathrm{eV}$ and the decreasing depletion at $285.2 \mathrm{eV}$. Further consideration of these intense field effects is beyond the scope of this discussion.

Sudden removal of an electron from a $\pi$ orbital by ionization changes the bonding pattern, initiating structural changes (JT distortion). ${ }^{56}$ The relaxation time depends on the slope of the potential energy surface (nuclear gradient) and distance between the initial FC geometry and the relaxed cation geometry. This is discussed in detail in Ref. ${ }^{56}$, where the time for structural relaxation was estimated to be on a timescale of 30 fs. Vibrationally excited states formed in the ionization process may affect the relaxation time, due to different potential energy surfaces for each vibration. ${ }^{57,26} \mathrm{As}$ noted above, there is room temperature internal energy in the neutral benzene (estimated average thermal vibrational energy) of about $0.05 \mathrm{eV}$ and $0.05 \mathrm{eV}$ of excess energy available from the twophoton UV excitation, which can prepare the benzene cation with a small amount of vibrational excitation, depending on the FC factors for the two-photon ionization from each neutral vibrational state. The spectrum of a pure vibrationless ground-state cation is therefore not expected in the experiment. There can be both vibrational redistribution after excitation, as well as relaxation from the FC region of the neutral to the structurally distorted cation, both of which can lead to significant changes in the $\mathrm{x}$-ray spectra and very early time dependences. ${ }^{58,59,60}$ Importantly, spectral signatures of the spin coupling of the unpaired electrons should be instantly visible upon ionization, as their appearance does not require structural changes and vibrational redistribution. Vibrational excitation alone may produce significant shifts in x-ray spectra, as illustrated in a recent study. ${ }^{58,60}$ In Figure 3, we present a time-dependent study that does show a trend of shifting of peaks E, F, G at different time delays during the period $0-150 \mathrm{fs}$.
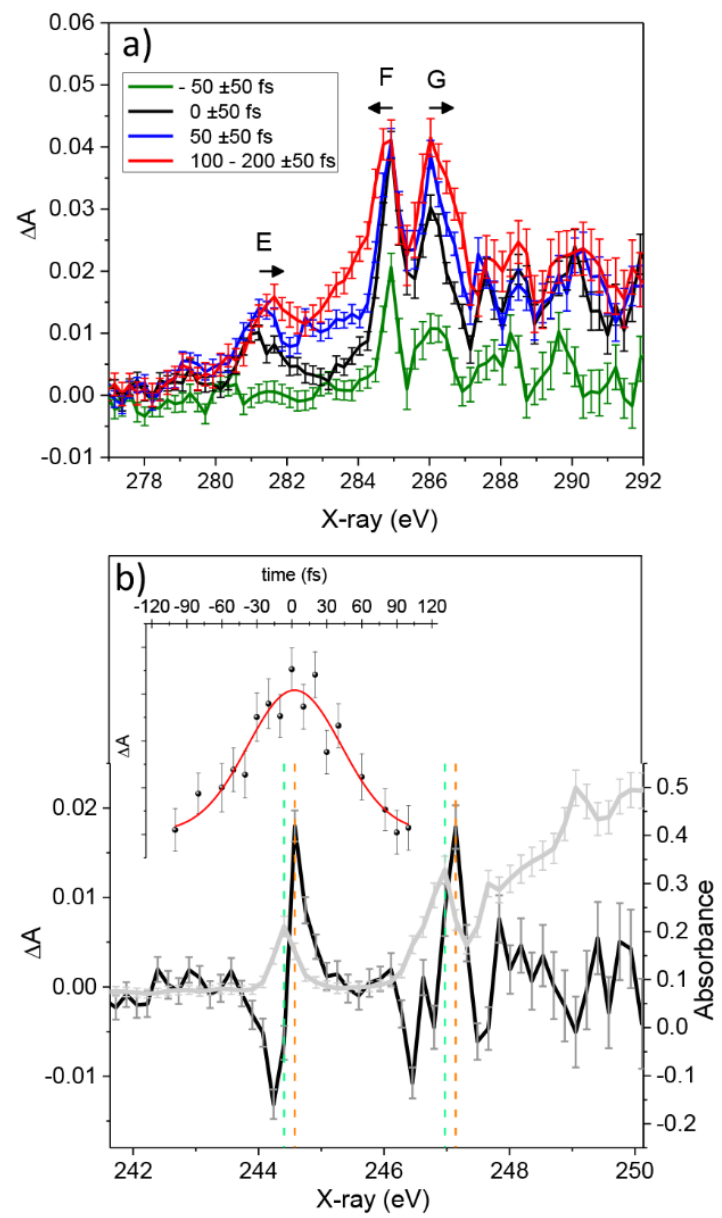

Figure 3. (a). Transient x-ray absorption spectra of ground state benzene cation measured at different time delays between the pump and probe beams. The black line represents $0 \pm 50$ fs delay where the probe and pump beam fully overlap (similarly to presented data in Figure 1(b)). The blue line represents $50 \pm 50 \mathrm{fs}$ and the red line represents $100-200 \pm 50$ fs delay. The experiment was performed with low intensity UV beam as can be seen from one partially open $\pi$ orbital indicated by only peak E. (b). Temporal resolution of the instrument measured by Stark shifting of XUV transitions of $\mathrm{Ar}$ with the UV pulse. In the top panel, the black dots represent the differential absorption $(\Delta \mathrm{A})$ spectrum of the $\operatorname{argon}{ }^{2} \mathrm{p}_{3 / 2} \rightarrow 4 \mathrm{~s}$ 
transition measured as a function of the pump (soft x-ray)-probe $(267 \mathrm{~nm})$ time delay (each spectrum is the average of $128 \mathrm{CCD}$ images, 1000 laser pulses per image) and the red line is a Gaussian fit with full-width-at-half-maximum (FWHM) of $90 \pm 15$ fs. The black line represents the differential absorption $(\Delta \mathrm{A})$ spectrum with the best temporal overlap (in this work defined as time zero) between $267 \mathrm{~nm}$ and x-ray radiation. The gray line represents the absorbance spectrum of argon without $267 \mathrm{~nm}$ laser to show the Stark shift of the Rydberg states (green and red dashed lines).

At longer time delays, peaks $\mathrm{E}$ and $\mathrm{G}$ move to higher energy $(\sim 0.2 \mathrm{eV})$ and peak F moves to lower energy $(\sim 0.3 \mathrm{eV})$, as indicated by the black arrows. The direction of the shifts follows what is expected from the JT displaced structure of the cation: the half-occupied $\pi$ orbital moves to higher energy and the two $\pi^{*}$ orbitals split to lower and higher energies relative to their position in the FC region. In addition to the observed peak shifts, the widths of the peaks change as well. In principle, the observed temporal dynamics should be related mostly to the JT displaced structure and vibrational redistribution, while spin coupling should be relatively insensitive to time delay and occur immediately. However, with the rather slow experimental temporal resolution of $90 \pm 15 \mathrm{fs}$, it is impossible to distinguish between the exact contributions from the structural and vibrational relaxation, versus exchange interactions due to spin splitting, or to define a precise timescale for the $\mathrm{x}$-ray spectral changes. This task would be further complicated if the spin coupling is also affected by nuclear motion, which could lead to a time-dependent behavior of the splitting energy due to the coupling of the three unpaired electrons.

Because the coupling of the three unpaired electrons is dominated by the valence exchange integral involving $\pi$ and $\pi^{*}$ orbitals, it may be sensitive to the exact shapes of the singly occupied valence orbitals. Therefore, the $1 \mathrm{sc} \rightarrow \pi^{*}$ transition may, in principle, report on changes in electron distribution along pseudo-rotation motion connecting two different JT electronic states of the cation differing by their singly occupied $\pi$ orbital. This might be an additional reason for observed trends in the peak widths.

It is also valuable to consider whether excitation to the high Rydberg states of neutral benzene by two-photon excitation near the ionization limit could lead to ultrafast relaxation to different electronic states of neutral benzene; ${ }^{61}$ this could be another reason for the broadening of the peaks. However, since the energy of the twophoton excitation is only slightly above the ionization limit, we consider this process as unlikely. Moreover, similar widths of the peaks are obtained even after tuning the UV wavelength in the 264 - $267 \mathrm{~nm}$ range by rotating the crystal that generates the third harmonic.

\section{CONCLUSION}

To conclude, this work investigates the carbon K-edge spectrum of the benzene radical cation following ionization with two UV photons. While many efforts have been directed to spectroscopic and theoretical investigations of the ground-state benzene radical cation and its JT distortion, the x-ray spectroscopic regime remained largely unexplored. The differences in the $\mathrm{x}$-ray spectra of the radical cation and the neutral benzene are directly compared in this contribution. In particular, the low-energy transition to the partially vacant $\pi$ orbital and the prominent splitting of the $1 \mathrm{sc}_{\mathrm{C}} \rightarrow \pi^{*}$ transition serve as clear fingerprints of the cation. The nature of this splitting is assigned to both the spin couplings of the three unpaired electrons (in the partially vacant $1 \mathrm{~s}$ core, the partially open $\pi$ orbital and the partly occupied $\pi^{*}$ orbital) and reduced symmetry of the cation following the JT distortion. The spectral changes in the first 100 fs do show clear shifts and broadenings in energy of the transitions involving $\pi$ and $\pi^{*}$ orbitals, which further deepens the understanding of the structural relaxation and the influence of spin coupling on the x-ray spectrum. More detailed analysis of the underlying electronic structure is discussed in the parallel theoretical paper. ${ }^{29}$ For a better understanding of the interplay between these two effects from an experimental perspective, measurements with attosecond or few-femtosecond time resolution are needed to distinguish the fast relaxation to the distorted cation symmetry from the instantaneous splitting due to spin coupling.

\section{ASSOCIATED CONTENT}

\section{Supporting Information}

X-ray transmission and absorption spectrum of neutral benzene; Calibration and spectral resolution of the experimental system; Initial population of vibrational states at room temperature; Pressuredependence study; Add-back correction for transient spectra. (PDF)

\section{AUTHOR INFORMATION}

\section{Corresponding Author \\ *srl@berkeley.edu}

\section{Present Addresses}

Michael Epshtein: Beer-Sheva, Israel 8471114

Zheyue Yang: No. 600 Cailun Road, Shanghai, China 201203

\section{ACKNOWLEDGMENT}

M.E., V.S., Z.Y., T.X., and S.R.L. gratefully acknowledge the generous support from the U.S. Department of Energy, Office of Science, Office of Basic Energy Sciences (Contract No. DEAC0205CH11231), the gas phase chemical physics program through the Chemical Sciences Division of Lawrence Berkeley National Laboratory.

V.S. acknowledges support from the Swiss National Science Foundation (P2ELP2_184414).

A.I.K. is a grateful recipient of the Simons Fellowship in Theoretical Physics and Mildred Dresselhaus Award from CFEL/DESY, which supported her sabbatical stay in Germany.

M.L.V. and S.C. acknowledge financial support from DTU Chemistry and from the Independent Research Fund Denmark-Natural Sciences, DFF-RP2 grant no. 7014-00258B. S.C. also thanks Prof. Piero Decleva (University of Trieste) for valuable discussions.

\section{REFERENCES}

(1) Spielmann, C.; Burnett, N. H.; Sartania, S.; Koppitsch, R.; Schnürer, M.; Kan, C.; Lenzner, M.; Wobrauschek, P.; Krausz, F. Generation of Coherent X-Rays in the Water Window Using 5-Femtosecond Laser Pulses. Science 1997, 278 (5338), 661-664.

(2) Popmintchev, T.; Chen, M.-C.; Arpin, P.; Murnane, M. M.; Kapteyn, H. C. The Attosecond Nonlinear Optics of Bright Coherent X-Ray Generation. Nat. Photonics 2010, 4 (12), 822-832.

(3) Seres, E.; Seres, J.; Krausz, F.; Spielmann, C. Generation of Coherent Soft-X-Ray Radiation Extending Far beyond the Titanium L Edge. Phys. Rev. Lett. 2004, 92 (16), 163002.

(4) Takahashi, E. J.; Kanai, T.; Ishikawa, K. L.; Nabekawa, Y.; Midorikawa, K. Coherent Water Window x Ray by Phase-Matched High-Order Harmonic Generation in Neutral Media. Phys. Rev. Lett. 2008, 101 (25), 253901 .

(5) Chen, M.-C.; Arpin, P.; Popmintchev, T.; Gerrity, M.; Zhang, B.; Seaberg, M.; Popmintchev, D.; Murnane, M. M.; Kapteyn, H. C. Bright, Coherent, Ultrafast Soft x-Ray Harmonics Spanning the Water Window from a Tabletop Light Source. Phys. Rev. Lett. 2010, 105 (17), 173901.

(6) Lou, B.; Ross, A. D.; Samay, G.; Kraus, P. M.; Neumark, D. M.; Leone, S. R. Efficient Table-Top Dual-Wavelength Beamline for Ultrafast Transient Absorption Spectroscopy in the Soft X-Ray Region. Sci. Rep. Nat. Publ. Group 2020, 10 (1). 
(7) Ishii, N.; Kaneshima, K.; Kitano, K.; Kanai, T.; Watanabe, S.; Itatani, J. Carrier-Envelope Phase-Dependent High Harmonic Generation in the Water Window Using Few-Cycle Infrared Pulses. Nat. Commun. 2014, 5 (1), 1-6.

(8) Cousin, S. L.; Silva, F.; Teichmann, S.; Hemmer, M.; Buades, B.; Biegert, J. High-Flux Table-Top Soft x-Ray Source Driven by Sub-2Cycle, CEP Stable, 1.85-mm 1-KHz Pulses for Carbon K-Edge Spectroscopy. Opt. Lett. 2014, 39 (18), 5383-5386.

(9) Silva, F.; Teichmann, S. M.; Cousin, S. L.; Hemmer, M.; Biegert, J. Spatiotemporal Isolation of Attosecond Soft X-Ray Pulses in the Water Window. Nat. Commun. 2015, 6 (1), 1-6.

(10) Li, J.; Ren, X.; Yin, Y.; Zhao, K.; Chew, A.; Cheng, Y.; Cunningham, E.; Wang, Y.; Hu, S.; Wu, Y. 53-Attosecond X-Ray Pulses Reach the Carbon K-Edge. Nat. Commun. 2017, 8 (1), 1-5.

(11) Kraus, P. M.; Zürch, M.; Cushing, S. K.; Neumark, D. M.; Leone, S. R. The Ultrafast X-Ray Spectroscopic Revolution in Chemical Dynamics. Nat. Rev. Chem. 2018, 2 (6), 82-94.

(12) Bhattacherjee, A.; Leone, S. R. Ultrafast X-Ray Transient Absorption Spectroscopy of Gas-Phase Photochemical Reactions: A New Universal Probe of Photoinduced Molecular Dynamics. Acc. Chem. Res. 2018, 51 (12), 3203-3211.

(13) Geneaux, R.; Marroux, H. J.; Guggenmos, A.; Neumark, D. M.; Leone, S. R. Transient Absorption Spectroscopy Using High Harmonic Generation: A Review of Ultrafast X-Ray Dynamics in Molecules and Solids. Philos. Trans. R. Soc. A 2019, 377 (2145), 20170463.

(14) Pertot, Y.; Schmidt, C.; Matthews, M.; Chauvet, A.; Huppert, M.; Svoboda, V.; Conta, A. von; Tehlar, A.; Baykusheva, D.; Wolf, J.-P.; Wörner, H. J. Time-Resolved x-Ray Absorption Spectroscopy with a Water Window High-Harmonic Source. Science 2017, 355 (6322), 264-267.

(15) Attar, A. R.; Bhattacherjee, A.; Pemmaraju, C. D.; Schnorr, K.; Closser, K. D.; Prendergast, D.; Leone, S. R. Femtosecond X-Ray Spectroscopy of an Electrocyclic Ring-Opening Reaction. Science 2017, 356 (6333), 54-59.

(16) Bhattacherjee, A.; Schnorr, K.; Oesterling, S.; Yang, Z.; Xue, T.; de Vivie-Riedle, R.; Leone, S. R. Photoinduced Heterocyclic Ring Opening of Furfural: Distinct Open-Chain Product Identification by Ultrafast X-Ray Transient Absorption Spectroscopy. J. Am. Chem. Soc. 2018, 140 (39), 12538-12544.

(17) Bhattacherjee, A.; Pemmaraju, C. D.; Schnorr, K.; Attar, A. R.; Leone, S. R. Ultrafast Intersystem Crossing in Acetylacetone via Femtosecond X-Ray Transient Absorption at the Carbon K-Edge. J. Am. Chem. Soc. 2017, 139 (46), 16576-16583.

(18) Yang, Z.; Schnorr, K.; Bhattacherjee, A.; Lefebvre, P.-L.; Epshtein, M.; Xue, T.; Stanton, J. F.; Leone, S. R. Electron-Withdrawing Effects in the Photodissociation of $\mathrm{CH}_{2} \mathrm{ICl}$ To Form $\mathrm{CH}_{2} \mathrm{Cl}$ Radical, Simultaneously Viewed Through the Carbon $\mathrm{K}$ and Chlorine $\mathrm{L}_{2,3} \mathrm{X}$-ray Edges. J. Am. Chem. Soc. 2018, 140 (41), 13360-13366.

(19) Schnorr, K.; Bhattacherjee, A.; Oosterbaan, K. J.; Delcey, M. G.; Yang, Z.; Xue, T.; Attar, A. R.; Chatterley, A. S.; Head-Gordon, M.; Leone, S. R. Tracing the 267 Nm-Induced Radical Formation in Dimethyl Disulfide Using Time-Resolved x-Ray Absorption Spectroscopy. J. Phys. Chem. Lett. 2019, 10 (6), 1382-1387.

(20) Loh, Z.-H.; Doumy, G.; Arnold, C.; Kjellsson, L.; Southworth, S. H.; Al Haddad, A.; Kumagai, Y.; Tu, M.-F.; Ho, P. J.; March, A. M. Observation of the Fastest Chemical Processes in the Radiolysis of Water. Science 2020, 367 (6474), 179-182.

(21) Kjellsson, L.; Nanda, K. D.; Rubensson, J.-E.; Doumy, G.; Southworth, S. H.; Ho, P. J.; March, A. M.; Al Haddad, A.; Kumagai, Y.; Tu, M.-F. Resonant Inelastic X-Ray Scattering Reveals Hidden Local Transitions of the Aqueous OH Radical. Phys. Rev. Lett. 2020, 124 (23), 236001.

(22) Raghavachari, K.; Haddon, R. C.; Miller, T. A.; Bondybey, V. E. Theoretical study of Jahn-Teller distortions in $\mathrm{C}_{6} \mathrm{H}_{6}{ }^{+}$and $\mathrm{C}_{6} \mathrm{~F}_{6}{ }^{+}$. J. Chem. Phys. 1983, 79 (3), 1387-1395.

(23) Bersuker, I. B. Modern Aspects of the Jahn- Teller Effect Theory and Applications to Molecular Problems. Chem. Rev. 2001, 101 (4), 1067 1114.

(24) Tachikawa, H. Jahn-Teller Effect of the Benzene Radical Cation: A Direct Ab Initio Molecular Dynamics Study. J. Phys. Chem. A 2018, 122 (16), 4121-4129.

(25) Lindner, R.; Müller-Dethlefs, K.; Wedum, E.; Haber, K.; Grant, E. R. On the Shape of $\mathrm{C}_{6} \mathrm{H}_{6}{ }^{+}$. Science 1996, 271 (5256), 1698-1702.

(26) Applegate, B. E.; Miller, T. A. Calculation of the Jahn-Teller Effect in Benzene Cation: Application to Spectral Analysis. J. Chem. Phys. 2002, 117 (23), 10654-10674.
(27) Nordfors, D.; Nilsson, A.; Mårtensson, N.; Svensson, S.; Gelius, U.; Lunell, S. Experimental and INDO/CI calculated gas phase C1s shakeup spectra of $\mathrm{C}_{6} \mathrm{H}_{6}, \mathrm{C}_{6} \mathrm{H}_{5} \mathrm{OH}$, and $\mathrm{C}_{6} \mathrm{H}_{5} \mathrm{CH}_{2} \mathrm{OH}$. J. Chem. Phys. 1988, 88 (4), 2630-2636.

(28) Sjögren, B. A Theoretical Study of the Shake-up Intensity of Benzene. J. Chem. Phys. 1992, 96 (11), 8338-8344.

(29) Vidal, M. L.; Epshtein, M.; Scutelnic, V.; Yang, Z.; Xue, T.; Leone, S. R.; Krylov, A. I.; Coriani, S. The Interplay of Open-Shell SpinCoupling and Jahn-Teller effect in Benzene Radical Cation Probed by Xray Spectroscopy. submitted to J. Phys. Chem. Lett, ChemRxiv link: doi $X X X X X$

(30) Lindblad, R.; Kjellsson, L.; Couto, R. C.; Timm, M.; Bülow, C.; Zamudio-Bayer, V.; Lundberg, M.; von Issendorff, B.; Lau, J. T.; Sorensen, S. L.; Carravetta, V.; Ågren, H.; Rubensson. J. E. X-Ray Absorption Spectrum of the $\mathrm{N}_{2}^{+}$Molecular Ion. Phys Rev Let. 2020, 124, 203001.

(31) Couto, R. C.; Kjellsson, L.; Ågren, H.; Carravetta, V.; Sorensen, S. L.; Kubin, M.; Bülow, C.; Timm, M.; Zamudio-Bayer, V.; von Issendorff, B. The Carbon and Oxygen K-Edge NEXAFS Spectra of $\mathrm{CO}^{+}$. Phys. Chem. Chem. Phys. 2020, 22 (28), 16215-16223.

(32) Hait, D.; Haugen, E. A.; Yang, Z.; Oosterbaan, K. J.; Leone, S. R.; Head-Gordon, M. Accurate Prediction of Core-Level Spectra of Radicals at Density Functional Theory Cost via Square Gradient Minimization and Recoupling of Mixed Configurations. ArXiv Prepr. ArXiv200610181 2020.

(33) Krylov, A. I. Triradicals. J. Phys. Chem. A 2005, 109 (47), 10638-10645

(34) Hitchcock, A. P.; Pocock, M.; Brion, C. E.; Banna, M. S.; Frost, D. C.; McDowell, C. A.; Wallbank, B. Inner Shell Excitation and Ionization of the Monohalobenzenes. J. Electron Spectrosc. Relat. Phenom. 1978, 13 (3), 345-360.

(35) Horsley, J. A.; Stöhr, J. +; Hitchcock, A. P.; Newbury, D. C.; Johnson, A. L.; Sette, F. Resonances in the K Shell Excitation Spectra of Benzene and Pyridine: Gas Phase, Solid, and Chemisorbed States. J. Chem. Phys. 1985, 83 (12), 6099-6107.

(36) Schwarz, W. H. E.; Chang, T. C.; Seeger, U.; Hwang, K. H. Core Excitations of Symmetrical Aromatic Molecules. Specific Correlations in the Valence Shell and Localization in the Core Shells. Chem. Phys. 1987, 117 (1), 73-89.

(37) Ma, Y.; Sette, F.; Meigs, G.; Modesti, S.; Chen, C. T. Breaking of Ground-State Symmetry in Core-Excited Ethylene and Benzene. Phys. Rev. Lett. 1989, 63 (19), 2044.

(38) Rennie, E. E.; Kempgens, B.; Köppe, H. M.; Hergenhahn, U.; Feldhaus, J.; Itchkawitz, B. S.; Kilcoyne, A. L. D.; Kivimäki, A.; Maier, K.; Piancastelli, M. N.; Polcik, M.; Rüdel, A.; Bradshaw, A. M. A comprehensive photoabsorption, photoionization, and shake-up excitation study of the C1s cross section of benzene. J. Chem. Phys. 2000, 113, 7362-7375.

(39) Kolczewski, C.; Martins, M.; Schlachter, A. S.; Snell, G.; Sant'Anna, M.; Viefhaus, J.; Hermann, K.; Kaindl, G. The C1s NEXAFS Spectrum of Benzene below Threshold: Rydberg or Valence Character of the Unoccupied $\sigma$-Type Orbitals. Chem. Phys. Lett. 2004, 393 (4), 361-366.

(40) Lunell, S.; Svensson, S.; Malmqvist, P. \AA; Gelius, U.; Basilier, E.; Siegbahn, K. A Theoretical and Experimental Study of the Carbon 1s Shake-up Structure of Benzene. Chem. Phys. Lett. 1978, 54 (3), 420 424.

(41) Myrseth, V.; Børve, K. J.; Wiesner, K.; Bässler, M.; Svensson, S.; Sæthre, L. J. Vibrational Structure and Vibronic Coupling in the Carbon 1s Photoelectron Spectra of Benzene and Deuterobenzene. Phys. Chem. Chem. Phys. 2002, 4 (24), 5937-5943.

(42) Nanda, K. D.; Vidal, M. L.; Faber, R.; Coriani S.; Krylov, A. I. How to stay out of trouble in RIXS calculations within equation-of-motion coupled-cluster damped response theory? Safe hitchhiking in the excitation manifold by means of core-valence separation. Phys. Chem. Chem. Phys. 2020, 22, 2629-2641. Ibidem (Erratum) Phys. Chem. Chem. Phys. https://doi.org/10.1039/D0CP90174A

(43) Asmuruf, F. A.; Besley, N. A. Time Dependent Density Functional Theory Study of the Near-Edge X-Ray Absorption Fine Structure of Benzene in Gas Phase and on Metal Surfaces. J. Chem. Phys. 2008, 129 (6), 064705 .

(44) Baltzer, P.; Karlsson, L.; Wannberg, B.; Öhrwall, G.; Holland, D. M. P.; MacDonald, M. A.; Hayes, M. A.; Von Niessen, W. An Experimental and Theoretical Study of the Valence Shell Photoelectron Spectrum of the Benzene Molecule. Chem. Phys. 1997, 224 (1), 95-119.

(45) Dawes, A.; Pascual, N.; Hoffmann, S. V.; Jones, N. C.; Mason, N. J. Vacuum Ultraviolet Photoabsorption Spectroscopy of Crystalline and 
Amorphous Benzene. Phys. Chem. Chem. Phys. 2017, 19 (40), 27544 27555 .

(46) Boesl, U.; Neusser, H. J.; Schlag, E. W. Visible and UV Multiphoton Ionization and Fragmentation of Polyatomic Molecules. J. Chem. Phys. 1980, 72 (8), 4327-4333.

(47) Chewter, L. A.; Sander, M.; Müller-Dethlefs, K.; Schlag, E. W. High Resolution Zero Kinetic Energy Photoelectron Spectroscopy of Benzene and Determination of the Ionization Potential. J. Chem. Phys. 1987, 86 (9), 4737-4744.

(48) Fally, S.; Carleer, M.; Vandaele, A. C. UV Fourier Transform Absorption Cross Sections of Benzene, Toluene, Meta-, Ortho-, and ParaXylene. J. Quant. Spectrosc. Radiat. Transf. 2009, 110 (9-10), 766-782.

(49) Etzkorn, T.; Klotz, B.; Sørensen, S.; Patroescu, I. V.; Barnes, I.; Becker, K. H.; Platt, U. Gas-Phase Absorption Cross Sections of 24 Monocyclic Aromatic Hydrocarbons in the UV and IR Spectral Ranges. Atmos. Environ. 1999, 33 (4), 525-540.

(50) Vidal, M. L.; Feng, X.; Epifanovsky, E.; Krylov, A. I.; Coriani, S. New and Efficient Equation-of-Motion Coupled-Cluster Framework for Core-Excited and Core Ionized States. J. Chem. Theory Comput. 2019, 15 , $5,3117-3133$

(51) Karlsson, L.; Mattsson, L.; Jadrny, R.; Bergmark, T.; Siegbahn, K. Valence Electron Spectra of Benzene and the Hexafluorides of Sulphur, Molybdenum, Tungsten and Uranium. An Application of Multichannel Detector Technique to UV-Valence Electron Spectroscopy. Phys. Scr. 1976, $14(5), 230$.

(52) Köppel, H. New ultrafast nonradiative decay mechanism in the benzene radical cation. Chem. Phys. Lett. 1993, 205, 361-370.

(53) Scheit, S.; Goswami, S.; Meyer, H.-D.; Köppel, H. Fully Quantal Treatment of Nonadiabatic Molecular Photodynamics: General Considerations and Application to the Benzene Cation. Comput. Theor. Chem 2019, 1150, 71-84.
(54) Galbraith, M. C. E.; Scheit, S.; Golubev, N. V.; Reitsma, G. Zhavoronkov, N.; Despré, V.; Lepine, F.; Kuleff, A. I.; Vrakking, M. J. J.; Kornilov, O. Few-Femtosecond Passage of Conical Intersections in the Benzene Cation. Nat. Commun. 2017, 8 (1), 1-7.

(55) Holland, D. M. P.; Shaw, D. A.; Sumner, I.; Bowler, M. A. Mackie, R. A.; Shpinkova, L. G.; Cooper, L.; Rennie, E. E.; Parker, J. E.; Johnson, C. A. F. A Time-of-Flight Mass Spectrometry Study of the Fragmentation of Valence Shell Ionised Benzene. Int. J. Mass Spectrom. 2002 220 (1), 31-51.

(56) Mendive-Tapia, D.; Vacher, M.; Bearpark, M. J.; Robb, M. A Coupled Electron-Nuclear Dynamics: Charge Migration and Charge Transfer Initiated near a Conical Intersection. J. Chem. Phys. 2013, 139 (4), 044110 .

(57) Johnson, P. M. The Jahn-Teller Effect in the Lower Electronic States of Benzene Cation. I. Calculation of Linear Parameters for the $e_{2 \mathrm{~g}}$ Modes. J. Chem. Phys. 2002, 117 (22), 9991-10000.

(58) Hosler, E. R.; Leone, S. R. Characterization of Vibrational Wave Packets by Core-Level High-Harmonic Transient Absorption Spectroscopy. Phys. Rev. A 2013, 88 (2), 023420.

(59) Kobayashi, Y.; Chang, K. F.; Zeng, T.; Neumark, D. M.; Leone, S. R. Direct Mapping of Curve-Crossing Dynamics in IBr by Attosecond Transient Absorption Spectroscopy. Science 2019, 365 (6448), 79-83.

(60) Kobayashi, Y.; Chang, K. F.; Poullain, S. M.; Scutelnic, V.; Zeng, T.; Neumark, D. M.; Leone, S. R. Coherent electronic-vibrational dynamics in deuterium bromide probed via attosecond transient absorption spectroscopy. Phys. Rev. A. 2020, 101, 063414

(61) Wiesenfeld, J. M.; Greene, B. I. Femtosecond Relaxation Dynamics of Molecular Rydberg States Using Time-Resolved Multiphoton Ionization. Phys. Rev. Lett. 1983, 51 (19), 1745. 


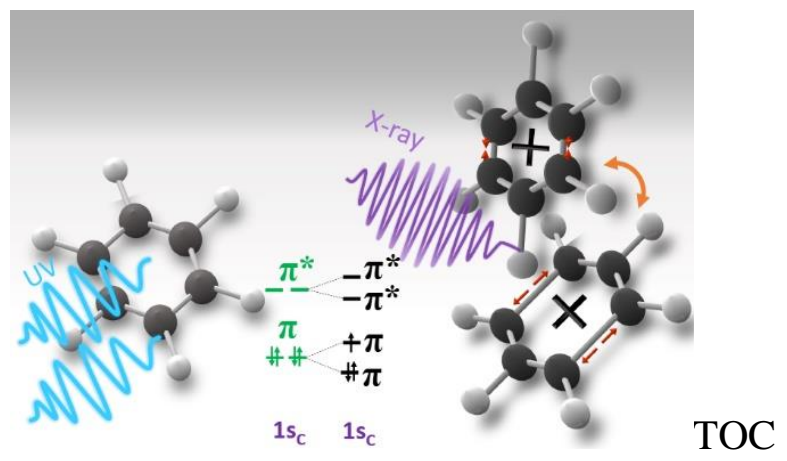

